\title{
Electrical Property Degradation and Mechanism of Aerospace White Paint Irradiated by Space Near UV Irradiation
}

\author{
Qiu Xiao-Lin ${ }^{1}$, Zhou Yi-Ren ${ }^{1, *}$ and Shen Zi-Cai ${ }^{2}$ \\ ${ }^{I}$ Nanchang Institute of Technology, No. 901 Heroe Ave, Qingshanhu, Nanchang, Jiangxi, 330044, China; ${ }^{2}$ Beijing Insti- \\ tute of Spacecraft Environment Engineering, Beijing 100094, China
}

\begin{abstract}
Aerospace white paint has excellent properties and can be sprayed in satellite antenna and other large spacecraft surfaces, while UV irradiation has important influence on the properties of it. The UV irradiation effect on the electrical properties of AEROSPACE white paint is studied firstly in this paper, and then the changing mechanism of it is discussed by the aid of XPS. It was found that $\mathrm{ZnO}$ in AEROSPACE white paint decomposes in ultraviolet irradiation and the color of AEROSPACE white paint turns to light yellow. The surface resistivity of AEROSPACE white paint decreases with near ultraviolet irradiation, and has back effect in some extent when it returns to atmosphere. Decomposition of $\mathrm{ZnO}$, decrease of adsorbed to Oxygen and increase of oxygen vacancy are the origin of decrease of surface resistivity of AEROSPACE white paint under near ultraviolet radiation.
\end{abstract}

Keywords: Electrical property, near ultraviolet, thermal control, white paint.

\section{INTRODUCTION}

The design of a spacecraft usually requires that its exterior surface has a low ratio of solar absorptance to emittance. This can be accomplished by applying suitable 'white' pigments blended with organic, semiorganic or inorganic binders to the exterior surface of the spacecraft $[1,2]$. Among the several thermal control coatings, AEROSPACE white paint, which is prepared by zinc oxide $(\mathrm{ZnO})$ blended with acryloid, has excellent properties and can be sprayed on satellite antenna and other large surfaces [3].

However, under the prolonged exposure to space radiation environment, the electrical properties and optical characteristics of it can be found to be degraded. For ultraviolet (UV) radiation is a major component of space radiation, it is necessary to study the degradation trend and mechanisms of AEROSPACE white paint, and these studies will have important applications in property prediction and improvement of thermal control coatings in the future.

The UV irradiation effects have been generally investigated only on the thin films and powders of $\mathrm{ZnO}$ [4]. Arun P. Kulshreshtha has reported UV irradiation effect on the electrical properties of $\mathrm{ZnO}$ thermal control coating pigment [1]. Feng Wei-quan et al. [5] have researched UV irradiation effect on some white paints, such as S781 and SR107-ZK, but without AEROSPACE white paint. Zeng Yibing has studied the space environmental and technical performances of AEROSPACE thermal control coatings, but only their solar absorptivity were researched.

In this study, UV irradiation effects on the electrical properties of AEROSPACE white paint are investigated, the degradation tendency and mechanisms of it are discussed. At last, the UV irradiation effects on the optical properties of AEROSPACE white paint are analyzed too.

\section{IRRADIATION TEST}

The test was performed in a low-energy combined environment test facility built by Beijing Institute of Spacecraft Environment Engineering (BISSE). This facility can provide environments of low-energy electrons, low-energy protons, NUV (near ultraviolet), FUV (far ultraviolet), or neutral plasma, thermal cycling and vacuum.

In the experiment, AEROSPACE white paints were irradiated by mercury xenon lamp with energy $1000 \mathrm{~W}$, the time for irradiation was long as 500ESH with low accelerator of 4 for real simulation, the working pressure was less than 10$3 \mathrm{~Pa}$, and the sample temperature was $30^{\circ} \mathrm{C}$. In order to simulate space effect, the surface resistivity of AEROSPACE white paint was measured in vacuum chamber by in-situ measuring system [6].

XPS was used to analyze the component of AEROSPACE white paint, and quadrupole mass spectrometer (QMS) was used to monitor the outgas of AEROSPACE white paint in near ultraviolet irradiation

\section{RESULTS AND ANALYSIS}

\subsection{Surface Color}

After irradiated by near ultraviolet, the color of AEROSPACE white paint changed from white to light yellow.

\subsection{Electrical Property}

The relation between irradiation time and surface resistivity of AEROSPACE white paint is firstly discussed. At 


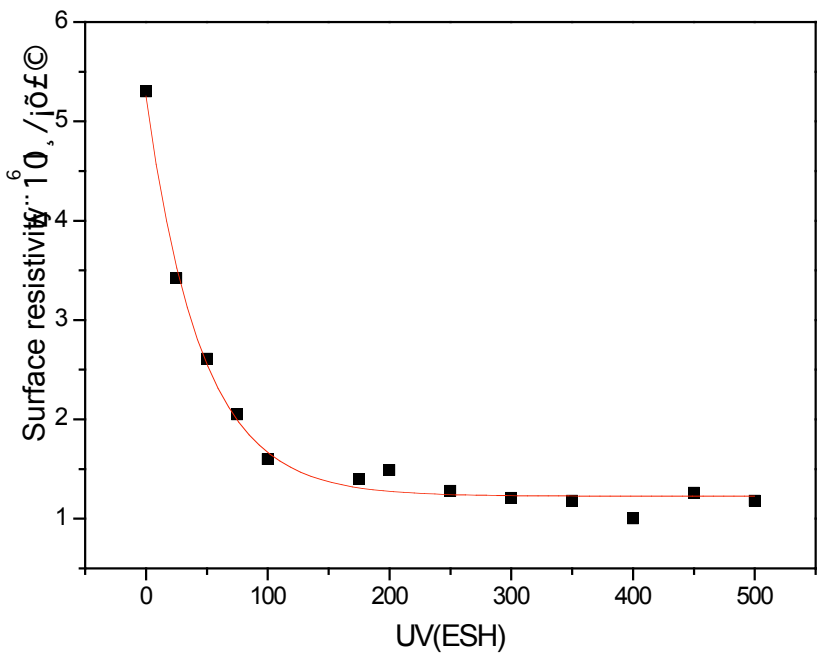

Fig. (1). Fitting surface resistivity versus irradiation time.

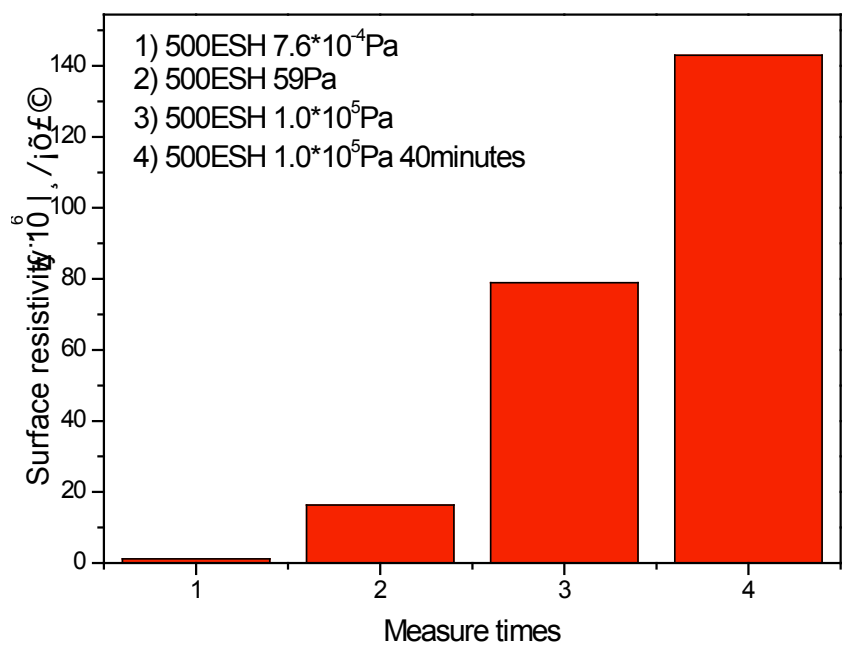

Fig. (2). Surface resistivity of AEROSPACE white paint versus pressure.

initial stages of UV irradiation within 25ESH, surface resistivity of AEROSPACE white paint decreases from $5.3 \times 109 \Omega / \mathrm{W}$ to $3.42 \times 106 \Omega / \mathrm{W}$. As the irradiation time prolongs, its surface resistivity decrease slowly, Fig. (1) shows the fitting result by:

$$
y=[1.226+4.028 \times \exp (-x / 45.225)] \times 10^{6} \Omega / \mathrm{W}(x \geq 25)
$$

\subsection{Vcauum Influence Analysis}

After being irradiated for 500ESH, the pressure of sample chamber, which AEROSPACE white paint was in, returned to atmosphere slowly, the surface resistivity of AEROSPACE white paint drastically increased, as shown in Fig. (2).

\section{MECHANISM STUDY}

The conductivity of AEROSPACE white paint is derived from $\mathrm{ZnO}$ pigment. $\mathrm{ZnO}$ is an n-type semiconductor and has intrinsic defect of oxygen vacancy, which is related with carrier density in it.
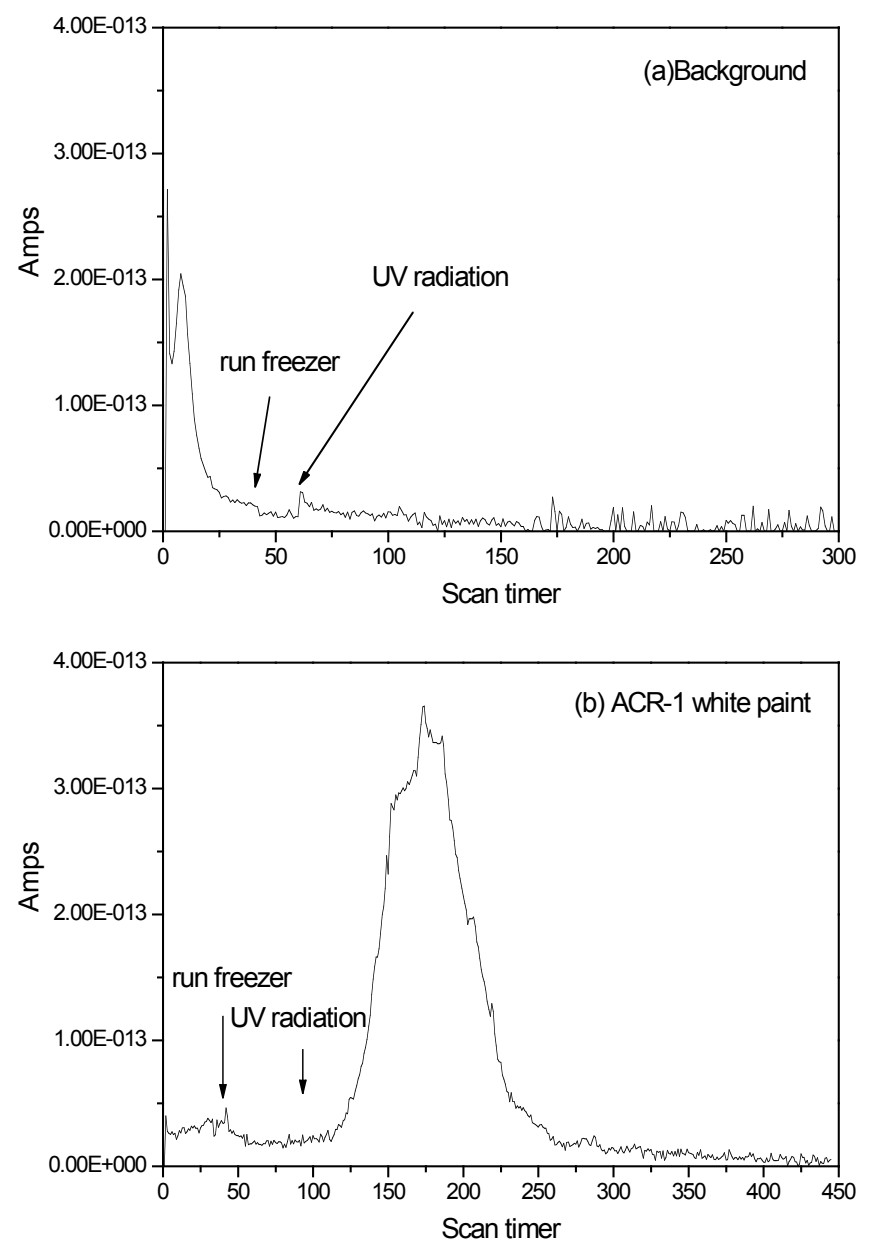

Fig. (3). Mass spectrum of $\mathrm{Zn}$ irradiated by near ultraviolet in Background or AEROSPACE white paint.

QMS was used to monitor the outgassing of AEROSPACE white paint in vacuum and ultraviolet irradiation, as shown in Fig. (3).

From Fig. (3), following conclusions can be obtained: As the beginning of near ultraviolet irradiation, $\mathrm{Zn}$ was released and AEROSPACE white paint turn to steady after be irradiated about 150 minutes.

Future analysis was confirmed by XPS, the Zn2p3 peak chart of AEROSPACE white paint, before and after irradiated by near ultraviolet, is shown in Fig. (4).

From Fig. (4), we know that 1021.82 is $\mathrm{Zn}$ and others are $\mathrm{ZnO}$, and the percentage of $\mathrm{Zn}$ increases and $\mathrm{ZnO}$ decreases.

$\mathrm{ZnO}$ in AEROSPACE white paint decomposes in ultraviolet irradiation, as follows:

$$
\mathrm{ZnO} \rightarrow \mathrm{Zn}+\frac{1}{2} \mathrm{O}_{2}, \mathrm{ZnO} \rightarrow \mathrm{Zn}_{i}+e^{\prime}+\frac{1}{2} \mathrm{O}_{2}
$$

X-ray photoelectron spectroscopy (XPS) was used to analysis the variety of oxygen in AEROSPACE white paint subjected to UV irradiation. Fig. (5) is the XPS of oxygen element prior and after exposure to 500ESH of UV irradiation. 

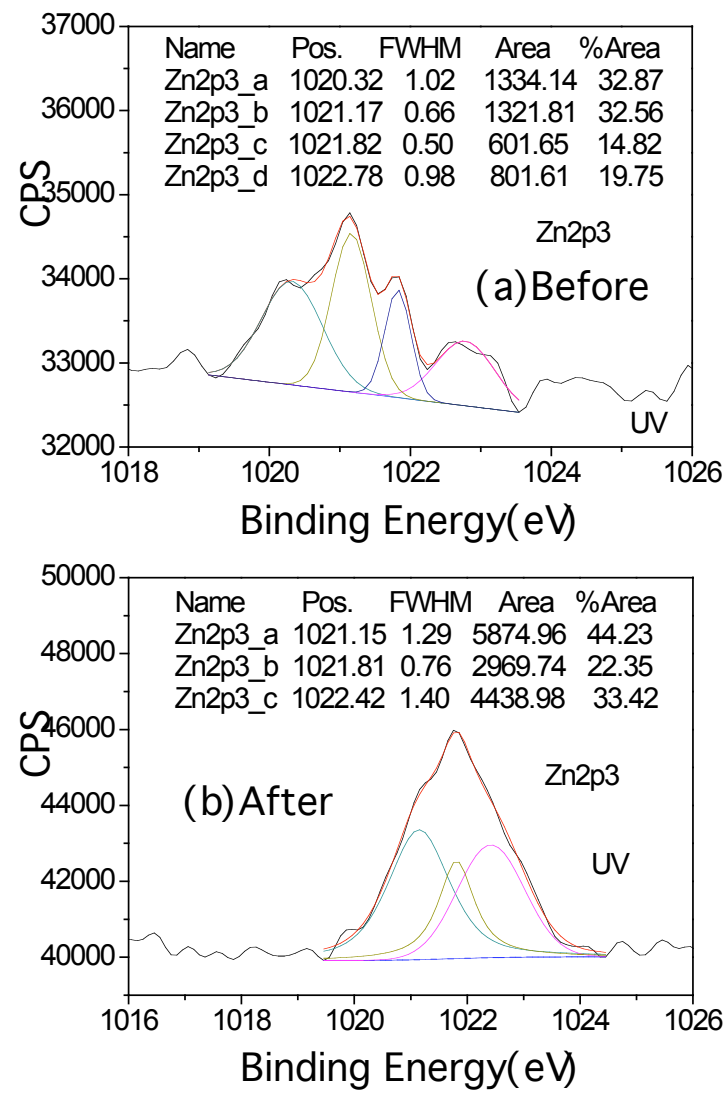

Fig. (4). Energy spectrum of Zn2p3 in AEROSPACE white paint before or after irradiated near UV irradiation.
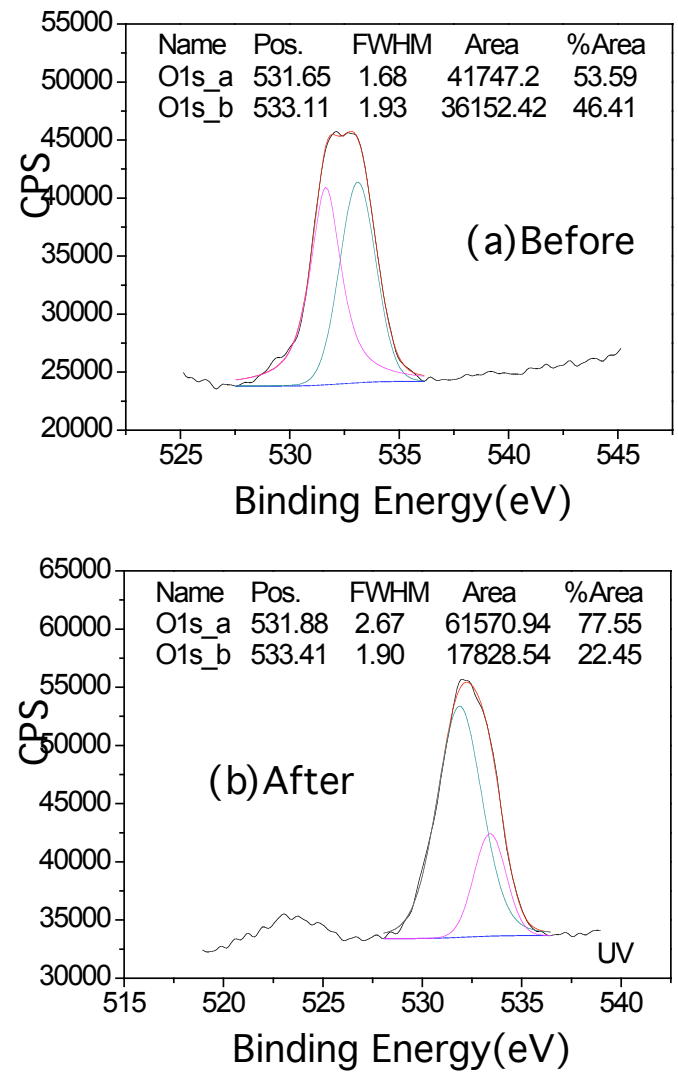

Fig. (5). XPS of O1s in AEROSPACE white paint before and after exposured to UV irradiation.
From Fig. (5), we know that there are at least two forms of oxygen in AEROSPACE white paint, one is adsorbed oxygen as O1s_a and another is chemical combined oxygen as $\mathrm{O} 1 \mathrm{~s}$ _b in spectroscopy $[7,8]$. Compared with two figures, it can be noticed that the adsorbed oxygen of AEROSPACE white paint decreases as the sample was irradiated.

Under normal conditions in air, zinc oxide is known to have chemisorbed oxygen on the surface. When subjected to $\mathrm{UV}$ irradiation in a space environment, $\mathrm{ZnO}$ in AEROSPACE white paint absorbs a higher percentage of incident solar energy, and the chemisorbed oxygen in it is released. Therefore, the improvement of conductivity or the decrease of surface resistivity of AEROSPACE white paint is due to the desorption of chemisorbed oxygen and due to the creation of oxygen ion vacancies by UV irradiation to some extent. As the oxygen pressure of AEROSPACE white paint returns to atmosphere, the oxygen will adsorbed to $\mathrm{ZnO}$ too, and this will result in the decrease of oxygen vacancy and increase of surface resistivity.

\section{SUMMARY}

$\mathrm{ZnO}$ in AEROSPACE white paint decomposes in ultraviolet irradiaton and the color of AEROSPACE white paint turns to light yellow. The surface resistivity of AEROSPACE white paint decreases with near ultraviolet irradiation, and has back effect to some extent when it returns to atmosphere. Decomposition of $\mathrm{ZnO}$, decrease of adsorb Oxygen and increase of oxygen vacancy are the origin of decrease of surface resistivity of AEROSPACE white paint under near ultraviolet radiation.

\section{CONFLICT OF INTEREST}

The authors confirm that this article content has no conflict of interest.

\section{ACKNOWLEDGEMENTS}

This research was financially supported by the National Natural Science Foundation of China (Grant No. 41174166).

\section{REFERENCES}

[1] A. P. Kulshreshtha, "UV irradiation effect on the electrical properties of $\mathrm{ZnO}$ thermal control coating pigment," IEEE Transaction on Aerospace and Electronic Systems AES-6, pp. 468-472, 1970.

[2] R. L. Kroes, and A. P. Kulshreshtha, "Effects of ultraviolet irradiation zinc oxide," AIAA, vol. 70-829, pp. 1-20, 2004.

[3] Y. B. Zeng, Z. P. Luo, Y. Li, "Space environmental and technical performances of antistatic wite thermal control coatings," Aerospace Materials \& Technology, vol. 37, pp. 21-24, 2007.

[4] J. D. Hayes and P. R. Mitchell, "Brown Engineering", Co. Inc. Rept. (1969) RL-int-632.

[5] W. Q. Feng, Y. G. Ding, and D. K. Yan, "The solar absorbance degradation property of thermal control coatings in nuv irradiation environment," SEE, vol. 20, pp. 13-18, 2003.

[6] GB1410-89 Methods of test for volume resistivity and surface resistivity of solid insulating materials, 1990. 
[7] C. F. John, and B, John, "X-ray photoemission spectroscopy studies of Sn-doped indium-oxide films," Journal of Applied Physics, vol. 48, pp. 3524-3531, 1977.
[8] M. Chen, Z. L. Pei, X. D. Bai, "XPS and AES Studies of ITO thin films," Chinese Journal of Materials Research, pp. 283-295, 2006.

Received: September 16, 2014

Revised: December 23, 2014

Accepted: December 31, 2014

(C) Xiao-Lin et al:; Licensee Bentham Open.

This is an open access article licensed under the terms of the Creative Commons Attribution Non-Commercial License (http://creativecommons.org/licenses/by-nc/3.0/) which permits unrestricted, non-commercial use, distribution and reproduction in any medium, provided the work is properly cited. 